\title{
Detectability of motions in AAA with ECG-gated CTA: A quantitative study
}

\author{
Almar Klein ${ }^{\text {a) }}$ \\ Institute of Technical Medicine, University of Twente, 7500 AE, Enschede, The Netherlands \\ Luuk J. Oostveen \\ Department of Radiology, Radboud University Nijmegen Medical Centre, 6525 GA, Nijmegen, \\ The Netherlands \\ Marcel J. W. Greuter \\ Department of Radiology, University Medical Center Groningen, 9700 RB, Groningen, The Netherlands \\ Yvonne Hoogeveen and Leo J. Schultze Kool \\ Department of Radiology, Radboud University Nijmegen Medical Centre, Nijmegen, The Netherlands \\ Cornelis H. Slump \\ Institute of Technical Medicine, University of Twente, Enschede, The Netherlands \\ W. Klaas Jan Renema \\ Department of Radiology, Radboud University Nijmegen Medical Centre, Nijmegen, The Netherlands
}

(Received 23 April 2009; revised 6 August 2009; accepted for publication 7 August 2009; published 14 September 2009)

Purpose: ECG-gated CT enables the visualization of motions caused by the beating of the heart. Although ECG gating is frequently used in cardiac CT imaging, this technique is also very promising for evaluating vessel wall motion of the aortic artery and the motions of (stent grafts inside) abdominal aortic aneurysms (AAA). Late stent graft failure is a serious complication in endovascular repair of aortic aneurysms. Better understanding of the motion characteristics of stent grafts will be beneficial for designing future devices. In addition, these data can be valuable in predicting stent graft failure in patients. To be able to reliably quantify the motion, however, it is of importance to know the performance and limitations of ECG gating, especially when the motions are small, as is the case in AAA. Since the details of the reconstruction algorithms are proprietary information on the CT manufacturers and not in the public domain, empirical experiments are required. The goal of this study is to investigate as to what extent the motions in AAA can be measured using ECG-gated CT. The authors quantitatively investigate four aspects of motion in ECG-gated CT: The detectability of the motion of objects at different amplitudes and different periodic motions, the temporal resolution, and the volume gaps that occur as a function of heart rate.

Methods: They designed an experiment on a standard static phantom to empirically determine temporal resolution. To investigate motion amplitude and frequency, as well as patient heart rate, they designed dynamic experiments in which a home-made phantom driven by a motion unit moves in a predetermined pattern.

Results: The duration of each ECG-gated phase was found to be $185 \mathrm{~ms}$, which corresponds to half of the rotation time and is thus in accordance with half scan reconstruction applied by the scanner. By using subpixel localization, motions become detectable from amplitudes of as small as $0.4 \mathrm{~mm}$ in the $x$ direction and $0.7 \mathrm{~mm}$ in the $z$ direction. With the rotation time used in this study, motions up to $2.7 \mathrm{~Hz}$ can be reliably detected. The reconstruction algorithm fills volume gaps with noisy data using interpolation, but objects within these gaps remain hidden.

Conclusions: This study gives insight into the possibilities and limitations for measuring small motions using ECG-gated CT. Application of the experimental method is not restricted to the CT scanner of a single manufacturer. From the results, they conclude that ECG-gated CTA is a suitable technique for studying the expected motions of the stent graft and vessel wall in AAA. (C) 2009 American Association of Physicists in Medicine.

[DOI: $10.1118 / 1.3213530]$

Key words: ECG gating, CTA, validation, motions, abdominal aortic aneurysm

\section{INTRODUCTION}

In recent years, there have been major advancements in computed tomography (CT). Shorter rotation times and the development of multidetector CT (MDCT) enabled the tech- nique of ECG gating. ${ }^{1}$ ECG gating uses the ECG signal of the patient to divide the raw scan data into bins that correspond to consecutive phases of the heart beat. The data are reconstructed into a number of volumes, each corresponding 
to a different phase of the heart cycle. This allows 4D visualization of the scanned object and enables the investigation of its temporal behavior. ${ }^{1,2}$

ECG gating is extensively used in cardiac exams, ${ }^{3-5}$ especially for the assessment of coronary arteries. ${ }^{6-8}$ The goal in most of these studies is to limit the effect of motion rather than to examine the motion itself for which the technique can also be utilized. Recently, ECG-gated CT angiography (CTA) was used to study the pulsating motion of abdominal aortic aneurysms $(\mathrm{AAA})^{9-11}$ and the motion of the renal arteries. ${ }^{12}$ Finally, ECG gating can also be used to evaluate the motion of implanted abdominal stent grafts. ${ }^{13}$

Late stent graft failure is a serious complication in endovascular repair of aortic aneurysms. ${ }^{14-17}$ Better understanding of the motion characteristics of stent grafts will be beneficial for designing future devices. In addition, these data can be valuable in predicting stent graft failure in patients. If detected, these patients will benefit from early reintervention.

The abdominal aorta is constantly in motion caused by the pressure waves from the contracting heart. However, the dynamics of this motion are more subtle than the motions present in the heart itself. To be able to reliably quantify these motions, it is of importance to know the capabilities and limitations of the applied ECG gating technique, especially when the motions of interest are small as in the case of AAA [of the order of $2 \mathrm{~mm}$ (Refs. 9 and 10)]. Several studies have been performed to validate the use of ECG gating for diagnostic purposes. ${ }^{3,4,6,7,18}$ Quantitative performance ${ }^{19}$ and simulation ${ }^{20}$ studies have also been performed. However, to the best of our knowledge, there are no quantitative studies on the performance of ECG-gated CTA with respect to the detectability of motions in AAA. Such a study is required to be able to distinguish measured motion from measurement errors in studies on motion using ECG-gated CTA and will help in designing future experiments to study motions of stent grafts in AAA.

The purpose of this paper is to investigate the performance of ECG gating quantitatively in motion detection for AAA. The results are compared to values that are theoretically determined on the basis of the scan parameters. This provides insight into the effects of the complex reconstruction algorithms - and the applied proprietary optimizations and corrections applied by manufacturers-on the motion detectability. Furthermore, the limits of the motion that can be detected in clinical practice by ECG gating is determined. In the present study, the research questions can be divided into four topics, which are discussed in the next sections.

\section{I.A. Temporal resolution}

In ECG-gated CT, temporal resolution consists of two parts (Fig. 1): The first is the width of each phase $T_{w}$, which is fully determined by the rotation time and reconstruction algorithm. Its value determines as to what extent motion causes artifacts in the resulting data. The second is the (temporal) distance between phases $T_{d}$, which is determined by

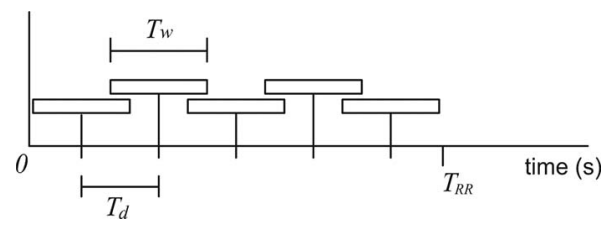

FIG. 1. Diagram illustrating the two aspects of temporal resolution. $T_{w}$ is determined by the rotation time and reconstruction algorithm. $T_{d}$ is determined by the heart rate $T_{R R}$ and the number of phases that we chose to reconstruct ( 5 in this example).

the number of phases and the heart rate. If more phases are reconstructed, then $T_{d}$ decreases and the overlap between phases increases.

Since using redundant data degrades the temporal resolution, ${ }^{21}$ optimal temporal resolution in terms of $T_{w}$ is achieved by minimizing the number of projections used to reconstruct the image. There are a variety of reconstruction algorithms, which result in different values for $T_{w}$. For standard fan beam reconstruction, for example, the minimum range of projections is $180^{\circ}$ plus the fan beam. ${ }^{21}$ For parallel beam reconstruction, however, temporal resolution of half of the rotation time can be achieved. ${ }^{1,2,20-22}$ Multisegment reconstruction can result in an even higher temporal resolution for some heart rates by reconstructing a volume using the raw data from different heart cycles. ${ }^{6,822,23}$ To be able to use an $N$-segment reconstruction, the spiral pitch factor (or pitch) has to be low enough and the heart rate high enough such that every $z$ location is imaged during at least $N$ heart beats. Because lowering the pitch generally results in a higher exposure, the technique can only be used at high heart rates.

Since $T_{w}$ depends on the applied reconstruction algorithm, which is often chosen by the manufacturer and of which the details are not in the public domain, an experiment was designed to determine it empirically.

\section{I.B. Amplitude}

The motions typically seen in (stent grafts inside) AAA are of the order of $2 \mathrm{~mm} .{ }^{9,10}$ The detectability of these motions depends on the localization accuracy of the object in each phase. The accuracy can be increased by using fitting techniques to find the noninteger (subpixel) location between two voxels of an object. However, the localization fit suffers from errors in the found location (localization noise), which can be larger than the motion itself if the motion's amplitude is low. We investigated the amplitude limit below which motions cannot be detected.

\section{I.C. Frequency}

The data collected during the time $T_{w}$ result in a single phase. Due to this averaging effect, there is an upper limit $f_{1}$ on the frequencies that can be measured. For a sinusoidal motion, this limit is 


$$
f_{1}=\frac{1}{2 T_{w}} .
$$

In order to measure motions accurately, a sufficient sampling rate is required (Nyquist frequency). This introduces a second upper limit $f_{2}$ for the measurable frequencies,

$$
f_{2}=\frac{1}{2 T_{d}}=\frac{N_{\text {phases }}}{2 T_{R R}}=\frac{N_{\text {phases }} \cdot B}{120},
$$

where $T_{R R}$ is the time of one heart cycle and $B$ is the beats per minute. Hereby is shown that patient's heart rate has a linear relation to the maximum frequency and, consequently, may affect the detectability of motion.

Motions in the abdominal aorta are produced by the pressure wave of the blood induced by the pumping of the heart. It has been shown that this pressure has a relatively simple shape: The pressure first increases quickly in around $200 \mathrm{~ms}$ and then decreases slowly until the next pressure wave. ${ }^{24-26}$ When the heart rate increases, the shape of the pressure increase is approximately constant.

In the present study, the aim is to determine which frequencies can be reliably detected before evident motion artifacts occur. To evaluate whether this is sufficient to reliably measure the motions as they occur in a clinical setting, the result is compared to the frequency components present in a pressure profile measured in vivo in the aortic artery, published in a study by Hazer et al. ${ }^{26}$ Additionally, we investigate whether certain motions, like motions synchronous with the rotation of the scanner, can result in unexpected behavior.

\section{I.D. Minimum required heart rate}

Figure 2 shows a diagram that illustrates the process of ECG gating. The dark patches represent a part of the phase in each heart beat. The overlap in the $z$ direction between these patches depends on the patient's heart rate. Increasing the number of phases will result in subsequent phases being closer together (in time), but two patches of the same phase will remain at equal distance (both in time as in the $z$ location).

In Fig. 2(b) it is shown that the time between two subsequent heart beats is too large for $40 \mathrm{bpm}$ : The $z$ coverage for two subsequent heart beats does not overlap, but shows a volume gap. To prevent this, the table displacement is limited to the nominal beam width during one heart cycle. The minimum heart rate $B_{\min }$ required to prevent volume gaps is given by $^{21,27}$

$$
B_{\min }=\frac{60 \cdot p}{T_{\text {rot }}},
$$

with $p$ as the pitch and $T_{\text {rot }}$ as the rotation time.

To lower the minimum heart rate, the pitch should be reduced, resulting in a longer scan time. Increasing the rotation time is not an option as it would increase motion blur (except for multisegment reconstruction at a certain heart rate). Equation (3) shows that if the rotation time is reduced, the pitch should be reduced accordingly. Since the number of photons that contribute to the reconstructed image depends
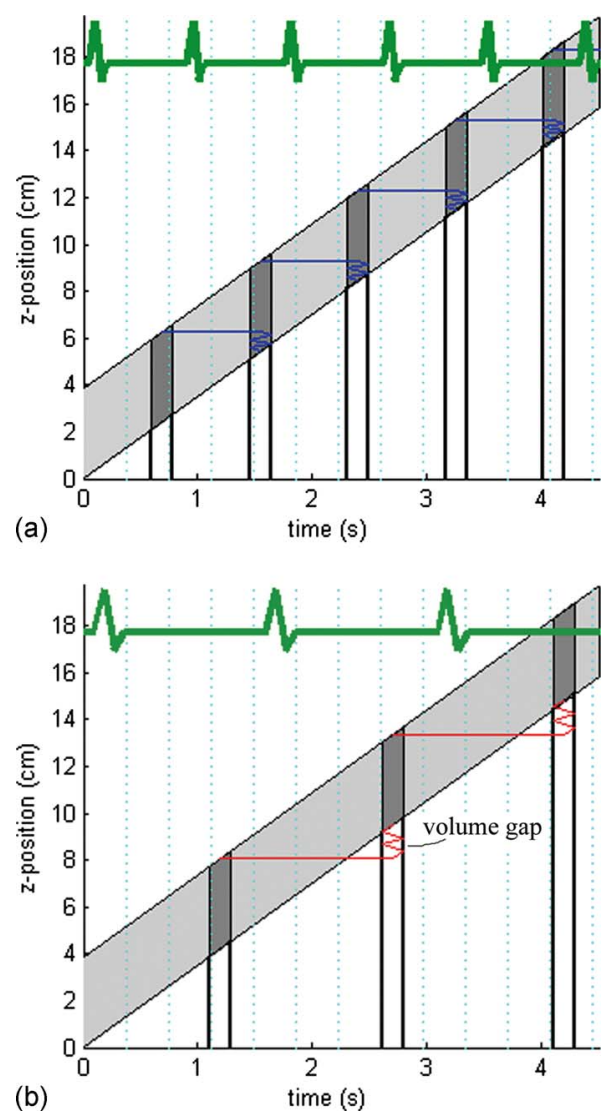

FIG. 2. Diagram illustrating the process of ECG gating. The light gray band indicates the covered $z$ positions of the detector during the scan. The dark gray patches represent parts of the phase in each heart beat. The horizontal lines that connect the patches indicate the measured $z$ position in subsequent parts of the same phase. They show the overlap [as in (a)] or volume gap [as in (b)] between the patches that belong to the same phase. The dotted vertical lines indicate the time at which the gantry is at $0^{\circ}$.

on the rotation time and the tube current only, decreasing the rotation time requires an increase in the tube current for the noise to remain the same. Because lowering the rotation time requires also lowering the pitch, the total exposure is increased. A faster rotation thus leads to a higher temporal resolution at the cost of increased exposure. ${ }^{20,21}$

It is of importance to verify the above theoretical limit and to know how the scanner performs in the presence of volume gaps since this can occur in clinical practice.

\section{MATERIALS AND METHODS}

All experiments were performed on a Siemens Somatom 64-slice CT scanner (Siemens Medical Solutions, Erlangen, Germany) with a rotation time of $0.37 \mathrm{~s}$, a pitch of 0.34 , and $2 \times 32 \times 0.6 \mathrm{~mm}^{2}$ collimation. An effective tube current time product of $180 \mathrm{mAs}$ was used at a tube voltage of $120 \mathrm{kVp}$.

\begin{tabular}{l|l|l|lll}
\hline $\mid$ & $\mid$ & $\mid$ & $\mid$ & $\mid$
\end{tabular}

FIG. 3. Schematic drawing of the phantom used for detecting motion. The phantom consists of a PMMA cylinder with stent wire fragments embedded at $20 \mathrm{~mm}$ intervals. 

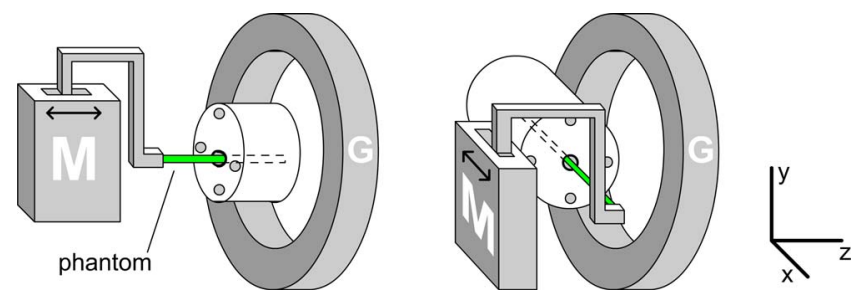

FIG. 4. Schematic drawing of the setup. The motion unit (M) drives the phantom (Fig. 3) inside the CTDI phantom's center hole, which is depicted in front of the gantry $(\mathrm{G})$. The left and right hand sides show the setup for measuring in the $z$ and $x$ directions, respectively.

The same parameters are used in the clinic, with the exception of the automated tube current modulation, which was turned off for our experiments. Retrospective gating was applied to obtain ten (equal distant) cardiac phases, unless stated otherwise. Each volume was reconstructed using the B36f reconstruction filter and resulted in approximately 80 slices of $512 \times 512$ voxels. The slices (thickness of $2 \mathrm{~mm}$ ) were spaced $1 \mathrm{~mm}$ apart, and the spacing between voxels in the $x y$ plane was approximately $0.5 \mathrm{~mm}$.

To quantitatively study motions in ECG-gated CT, a device capable of moving in a predetermined pattern was used (PC Controlled Phantom Device, QRM, Möhrendorf, Germany). It consists of a motion unit that moves a lever to which a phantom can be attached. The phantom (constructed in-house) consisted of a cylinder made of PMMA (length of $160 \mathrm{~mm}$, diameter of $10 \mathrm{~mm}$ ) in which pieces of nitinol wire were embedded at $20 \mathrm{~mm}$ intervals (Fig. 3). The wires (length of approximately $6 \mathrm{~mm}$ and diameter of $0.2 \mathrm{~mm}$ ) were cut from the framework of a stent graft and resulted in highly localized points (with a full width at half maximum of approximately $2-3$ voxels in the $x y$ plane). A standard CTDI body phantom (32 $\mathrm{cm}$ in diameter) was used to provide a tissuelike medium and functioned as a guide for the cylindrical phantom to move in (Fig. 4). To drive the motion unit, seven amplitude patterns and seven frequency patterns were designed (Table I). Triangular motion patterns were used such that the resulting motion was linear and could easily be mathematically described. No assumptions about the shape of the motion were made since the goal of the present study was to investigate the motion's frequency components individually. To realize a higher frequency, some patterns consisted of multiple triangular periods per cardiac phase. An ECG signal was provided by the motion unit during the scan.

Using profiles A0-A6 (Table I), measurements of the motion amplitude were performed. Note that with amplitude, we refer to the peak-to-peak value of the motion. For each profile, one scan was performed in the $x$ direction and one in the $z$ direction (Fig. 4). The detectability in the $x$ and $y$ directions can be assumed equal due to the scanner geometry. To quantify the effects of the amplitude on the detectability of motion, the bright spots where the nitinol wires penetrate the slice are detected. Next, a triangular shape with the appropriate amplitude is fitted through the points, and the localization errors of the detected points are calculated by subtracting the fitted triangle from the found locations.

Profiles B0-B6 were designed to investigate the frequency characteristics and were set up to go from low to above the expected maximum measurable frequency. Profiles B0-B3 have a $T_{R R}$ around the expected minimum required heart rate, which is [according to Eq. (3)] 55.1 beats/min. Profile B4 is designed to move synchronous with the rotation of the scanner. All measurements with profiles B0-B6 were done in the $z$ direction because of practical considerations concerning the setup. To measure the detectability of motion as a function of frequency, the same approach was used as for the amplitude measurements. To be able to compare the results of the frequency measurements with the frequency characteristics present in a clinical setting, the reported pressure profile published by Hazer $e t$ al. $^{26}$ was used. The spectrum of the profile was obtained using the fast Fourier transform.

To measure the temporal resolution $\left(T_{w}\right)$, the uniform module of the Catphan phantom (The Phantom Laboratory, Salem, USA) was scanned with ECG gating using the simulation ECG signal of the scanner at $70 \mathrm{bpm}$. This single scan was then reconstructed eight times with the number of phases ranging from 3 to 10 . For three phases, there was no overlap between two subsequent phases. For higher number of phases, the overlap between two subsequent phases increases. We measured the correlation coefficient for a set of

TABLE I. The motion patterns used in the experiments. A0-A6 vary in amplitude, while B0-B6 vary in frequency.

\begin{tabular}{cccccccc}
\hline \hline & A0 & A1 & A2 & A3 & A4 & A5 & A6 \\
\hline No. of periods per phase & 1 & 1 & 1 & 1 & 1 & 1 & 1 \\
Heart rate & 60 & 60 & 60 & 60 & 60 & 60 & 60 \\
Frequency (Hz) & 1.0 & 1.0 & 1.0 & 1.0 & 1.0 & 1.0 & 1.0 \\
Amplitude (mm) & 0.2 & 0.4 & 0.7 & 1.2 & 2.0 & 3.0 & 4.0 \\
& B0 & B1 & B2 & B3 & B4 & B5 & B6 \\
\hline No. of periods per phase & 2 & 2 & 2 & 2 & 3 & 3 & 3 \\
Heart rate & 45 & 54 & 56 & 60 & 54.05 & 60 & 80 \\
Frequency (Hz) & 1.5 & 1.8 & 1.87 & 2.0 & 2.7 & 3.0 & 4.0 \\
Amplitude (mm) & 3.0 & 3.0 & 3.0 & 3.0 & 3.0 & 3.0 & 3.0 \\
\hline \hline
\end{tabular}


voxels in two subsequent phases: $\rho_{a, b}=E\left(\left(A-\mu_{a}\right)(B\right.$ $\left.\left.-\mu_{b}\right)\right) /\left(\sigma_{a} \sigma_{b}\right)$, with $E$ as the expected value operator, $A$ and $B$ as the voxel data of the two phases, $\mu$ as the mean, and $\sigma$ as the standard deviation. The resulting number (between 0 and 1) indicates as-to what extent the noise is correlated (i.e., coming from the same source) and is a measure for the overlap between the two phases. The point at which there is just no overlap between subsequent phases is the point where $T_{d}$ and $T_{w}$ are equal. Estimating this point gives us $T_{w}$.

We developed algorithms in Python (an open source programming language, www.python.org) to process the data on a PC. To process the results of the moving phantom scans, the slices penetrated by the nitinol wires in the phantom were manually selected. To compensate for the noise in scans in which motion in the $z$ direction was measured, multiple slices were averaged. Next, the locations of the stent graft wires in the phantom were automatically detected by finding the voxel with maximum intensity in a region where the wire is expected, and the subpixel location is estimated using a polynomial fit. Would a 2D quadratic fit be used, the system of equations is overdetermined (nine equations and five unknowns) and the result would be a least squares solution, which is noninterpolating and can therefore deviate more than half a pixel from the detected integer location. Therefore, two 1D quadratic polynomials were used to fit the $x$ and $y$ subpixel locations independently.

\section{RESULTS}

To measure $T_{w}$, the overlap of the different phases was determined from the correlation of the noise. The correlation between two subsequent phases is shown in Fig. 5. For a low number of phases, the correlation is 0 . From the point where $T_{w}$ equals $T_{d}$, the correlation increases as the number of phases increases. It can be seen in Fig. 5 that this occurs after approximately four phases (for 70 beats/min). The dotted line represents the correlation of two phases that are separated by one phase, in which case the correlation starts to rise after 8 or 9 phases. In Fig. 5(b) the correlation is plotted as a function of the temporal distance $T_{d}$. The lines incline in a linear fashion, which enables fitting a line through the points and finding the point (on the $x$ axis) where $T_{d}$ is equal to $T_{w}$. The dashed line shows the fit, which intersects with zero correlation at $T_{d}=186.6 \pm 2.4 \mathrm{~ms}$, which corresponds to half of the rotation time as used in the experiment. Hence the exact value of $T_{w}$ can be assumed to be $185 \mathrm{~ms}$.

The motion detectability was derived from the measured positions (with the mean subtracted) of the detected points and is shown for different amplitudes in Fig. 6. The scans contain four or more points in each of the ten phases, resulting in at least 40 data points per scan. The triangular shape becomes more apparent as the amplitude increases. The absolute error as a function of amplitude (after subtraction of the known triangular shape) is illustrated in Fig. 7.

To determine which frequencies can be reliably detected, the absolute error was calculated for different frequencies (Fig. 8). Figure 9 shows an example of a detected motion for profile B5 $(3 \mathrm{~Hz})$. In Sec. I, we discuss the possibility of
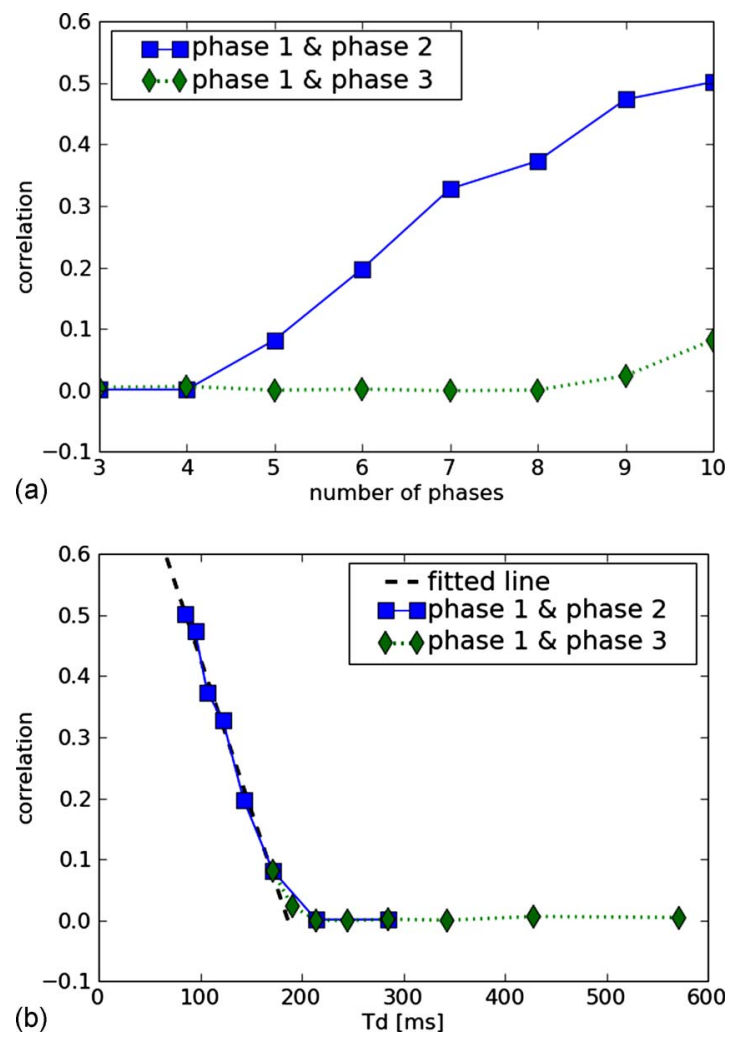

FIG. 5. Illustration of the correlation between subsequent phases against the number of phases (a) and against the time between phases (b). The dashed line in (b) is a linear fit through the seven data points left of the $180 \mathrm{~ms}$ mark.

unexpected results for motions synchronized with the gantry rotation. This was investigated (using the scan with motion profile B4), but no differences compared to the other scans were detected. Figure 10 shows the shape and spectrum of a pressure profile measured in vivo in the aortic artery. It can be seen that the spectrum contains several higher harmonics.

The minimum required heart rate was determined by examining four slices through the phantom at heart rates around the minimum required theoretical heart rate of $55.1 \mathrm{bpm}$. Figure 11 illustrates four example slices at profiles B0-B3. From the lowest heart rate in Fig. 11, one can clearly observe the noisy bands due to the volume gap, which propagate from top to bottom for increasing phase number. At $54 \mathrm{bpm}$ the bands are still visible, but very thin. For $56 \mathrm{bpm}$, which is just above the theoretical limit, a band can be observed in some phases (near the top of the shown image, for example) on close examination. For $60 \mathrm{bpm}$, however, the images contain no noise bands. In Fig. 11(a) four bars of the phantom can be seen, of which the first, third and fourth from the top are clearly visible. The second, however, seems to have disappeared, while it is clearly visible in the other phases and in the other examples.

\section{DISCUSSION}

In the current study, several experiments were performed to evaluate the temporal resolution, the effect of amplitude, and frequency on the detectability of motion, and the minimum heart rate. 

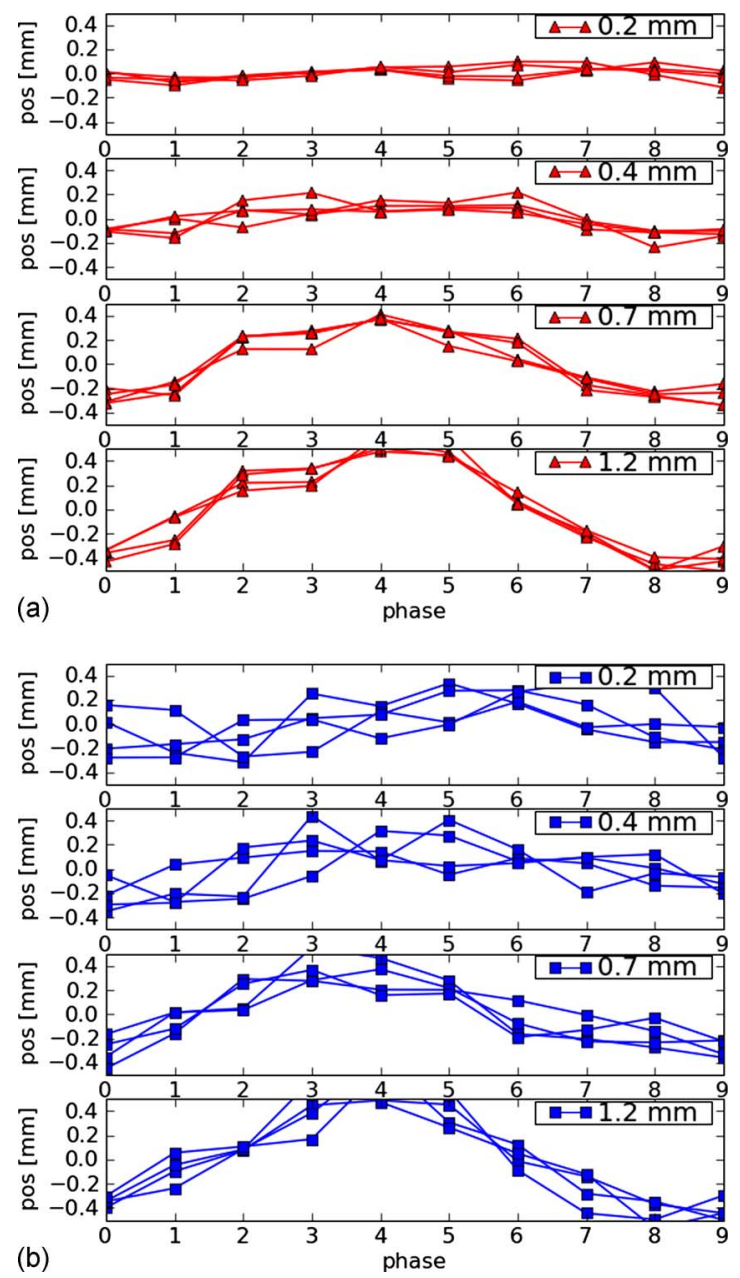

FIG. 6. Illustration of the moving position of the points for different amplitudes.

\section{IV.A. Temporal resolution}

The value of $T_{w}$ was found to be $185 \mathrm{~ms}$, which corresponds to half of the rotation time. This result strongly suggests that the scanner used a half scan reconstruction algorithm. However, above a certain heart rate some scanners might switch to multi segment reconstruction, which results in higher temporal resolution. ${ }^{8,23}$

The number of phases to reconstruct should be chosen such that there is overlap between subsequent phases $\left(T_{d}\right.$ $<T_{w}$ ) even for patients with low heart rates. For our settings and a heart rate of $50 \mathrm{bpm}\left(T_{R R}=1.2 \mathrm{~s}\right)$ this is $1.2 / 0.185=7$ phases. Using more phases results in a higher temporal resolution (in terms of $T_{d}$ ). However, because more than $50 \%$ overlap between subsequent phases results in redundant data, a maximum number can also be calculated: For a heart rate of $50 \mathrm{bmp}$ this is achieved at $2 \times 1.2 / 0.185=13$ phases. We can thus conclude that for ECG gating (on our scanner type) using $8-12$ phases is a reasonable choice.

The described experiment enables measuring the temporal resolution in a generic and reliable way, is applicable to other scanner types, and can be performed on any phantom with a uniform volume.
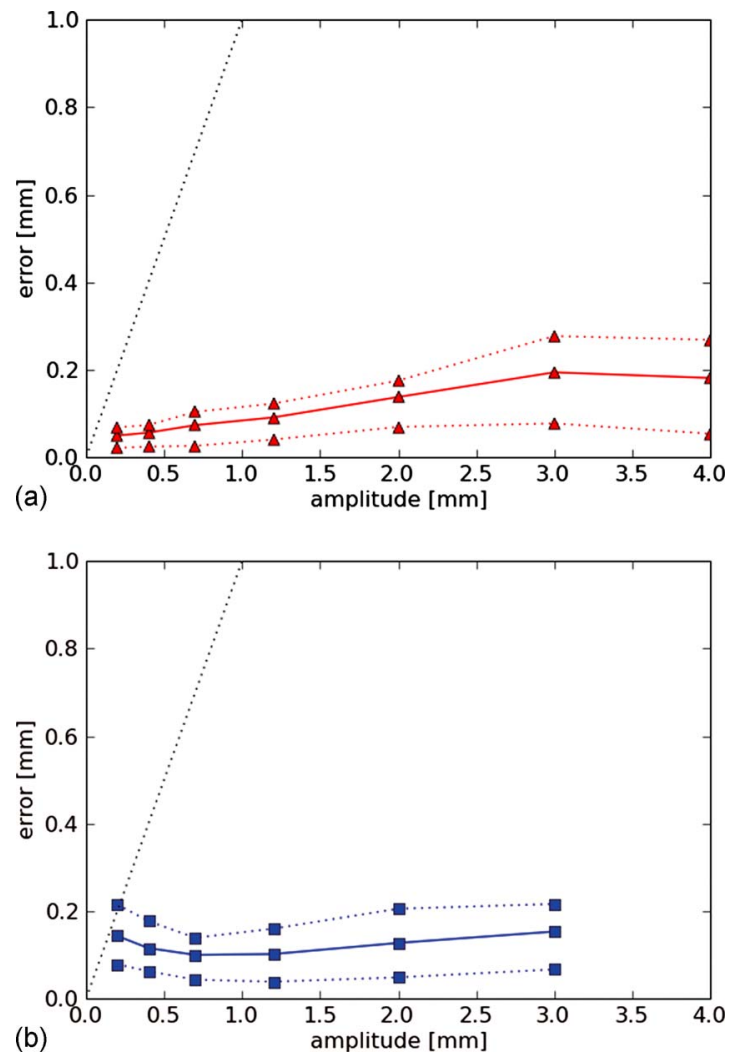

FIG. 7. Illustration of the error versus amplitude. The solid line represents the mean absolute error. The dotted lines are the 25 and 75 percentile of the sorted absolute error of the $40+$ data points in each experiment. The dotted $45^{\circ}$ line indicates where the error and amplitude are equal.

\section{IV.B. Amplitude}

Figures 6 and 7 show that, as expected, the error in localization is higher in the $z$ direction because the voxel size is approximately twice as large as in the $x$ direction $(1.0 \mathrm{~mm}$ versus approximately $0.5 \mathrm{~mm}$ ). From Fig. 7 it can be seen that, as anticipated, the error is nearly constant. The slight slope is probably due to the effect of motion artifacts, which become more prominent as the amplitudes increases. In Figs. $7(\mathrm{a})$ and 7(b) the amplitude exceeds the noise level when the

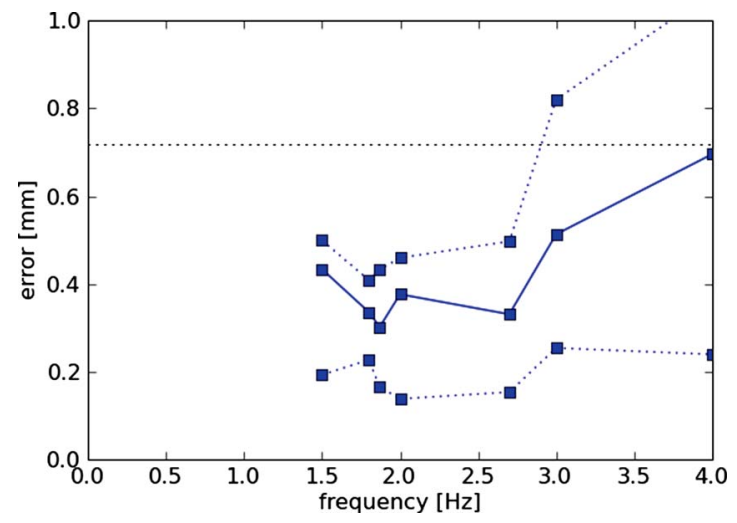

FIG. 8. Illustration of the error versus frequency. The solid line represents the mean absolute error. The dotted lines are the 25 and 75 percentile of the sorted absolute error. 


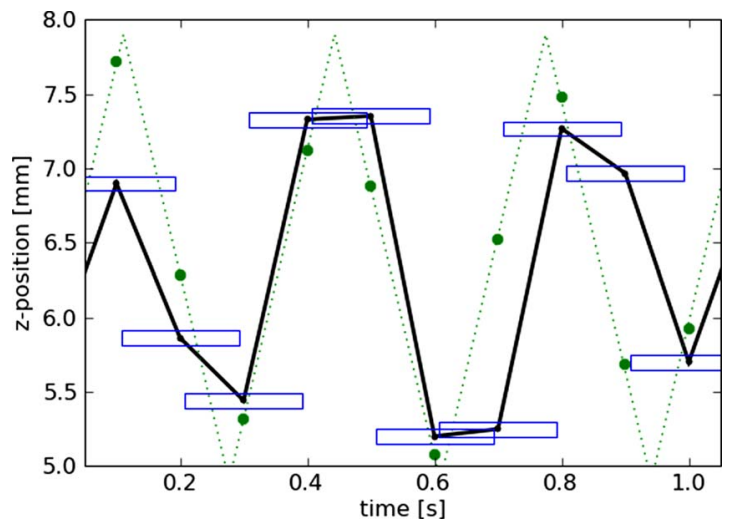

FIG. 9. Example of the detected motion (solid) of a point at $3.0 \mathrm{~Hz}$ and the known profile (dotted). The horizontal boxes indicate the temporal width $T_{w}$ of $185 \mathrm{~ms}$.

error is to the right of the dotted $45^{\circ}$ line. Naturally, this is not an abrupt process: The motion will emerge from the noise with increasing amplitude. Nonetheless, from Fig. 6 it can be seen that amplitudes as small as $0.4 \mathrm{~mm}$ in the $x$ direction and $0.7 \mathrm{~mm}$ in the $z$ direction can be detected.

In the experiments for the amplitude measurements, it is of importance that the phantom moves accurately according to the intended profile. Two sources of error can be distinguished. First, the motion unit. According to its specification, the precision of the start position of the motion unit is better than $0.2 \mathrm{~mm}$ and the reproducibility of motion profiles in quasistationary state better than $1 \%$. This suggests that the device may introduce small errors for the lower amplitudes. Second, the transfer of the motion to the phantom. In the $z$ direction this transfer was realized by directly attaching the phantom to the lever. The lever was then fixed in such a position (using bolts) that the phantom moved smoothly in its guide. Due to mechanical restrictions for the $x$ direction, however, the motion had to be transferred via a corner piece, which allows some minor bending. By ensuring the phantom moved smoothly in its guide, the friction was reduced as much as possible. The fact that the accuracy was found to be better in the $x$ direction suggests that the measurements were not unduly affected by the latter source of possible error.

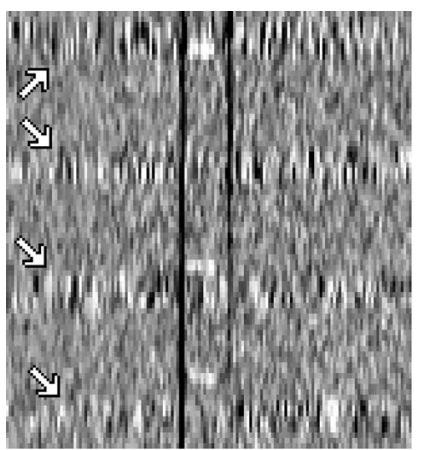

(a)

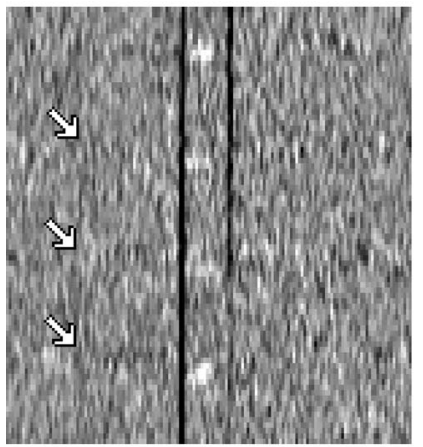

(b)

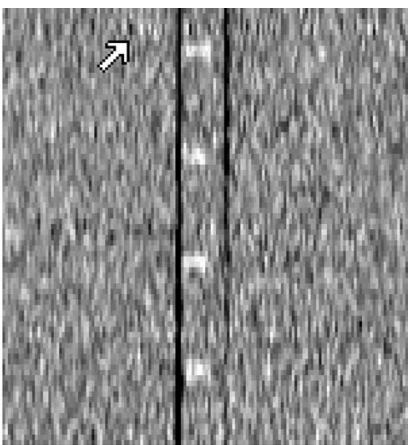

(c)

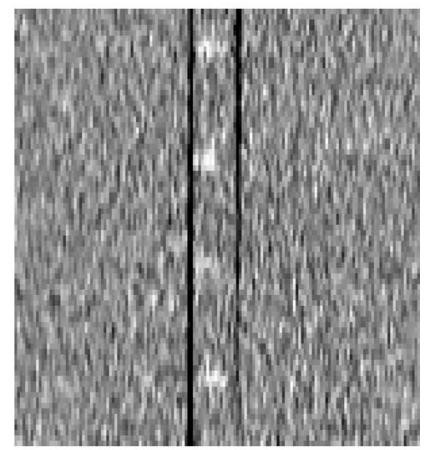

(d)

FIG. 11. Illustration of the noise bands in the CT images caused by the volume gaps due to a too low heart rate during scanning. At $45 \mathrm{bpm}$, the (horizontal) noise bands are clearly visible (indicated by the arrows). It can be seen how it hides the second bar from the top. At $54 \mathrm{bpm}$, the noise bands are very thin. At higher heart rates, no noise bands can be detected.

Noise in the image data causes errors in the subpixel localization. Thus it is expected that when images with less noise would be produced, the accuracy will increase. However, to realize the latter, exposure will have to be increased. In our experiments we used an exposure comparable with that used in the clinical setting.

\section{IV.C. Frequency}

The B0 measurement $(1.5 \mathrm{~Hz})$ had a relatively large error due to the heart rate that was too low during this measure-
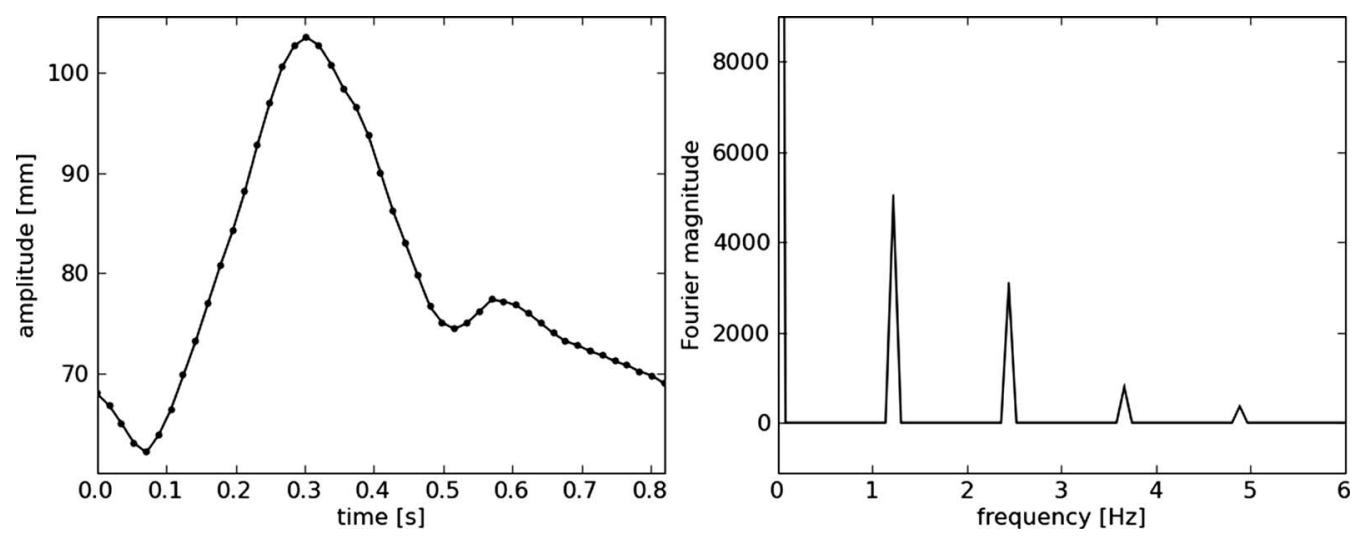

FIG. 10. Illustration of the shape (left) and the Fourier response (right) of a pressure profile in the aortic artery, as reported in literature (Ref. 26). 
ment (Fig. 8). The next three measurements had a relatively low error, and from $2.7 \mathrm{~Hz}$ and up the error increases.

With ten phases and a heart rate of $50 \mathrm{bpm}, T_{d}=120 \mathrm{~ms}$ and the Nyquist frequency is found at $4.2 \mathrm{~Hz}$ [Eq. (2)], which is well above the frequencies examined. Since higher heart rates give rise to even higher Nyquist frequencies, the Nyquist criterion is of minor importance for our experiments.

Due to the temporal width of the phases, the effect of motion artifacts increases with increasing frequency, until they reach an upper limit, above which the motion should not be measurable. Given $T_{w}=185 \mathrm{~ms}$, the upper limit is found at $2.7 \mathrm{~Hz}$ [Eq. (1)]. However, the results suggest that motion at 2.7 can be measured relatively well. To study this in more detail, the detected motion of a point at $3 \mathrm{~Hz}$ is shown in Fig. 9 , indicating the temporal phase width $T_{w}$ using horizontal boxes of $185 \mathrm{~ms}$. (Half scan reconstruction was assumed, as the bpm in this measurement was lower than that used in the temporal resolution experiment.) As $185 \mathrm{~ms}$ is over half of the period of motion $(167 \mathrm{~ms})$, we would expect the estimated motion to be poor, yet (for most points) the estimated locations are good. This surprising result can be explained by the way the data-acquired during half a rotation-is processed; because of the reconstruction, motion during the acquisition results in highly localized motion artifacts and not necessarily blurring. Therefore, the location of the detected point can still be relatively accurate.

Figure 10 shows that the pressure (and thus the motion) in the aortic artery contains frequency components higher than $2.7 \mathrm{~Hz}$. This will express itself in motion artifacts in the phases acquired during the sudden rise of pressure at the start of the cardiac cycle. Consequently, these phases can be rendered useless if the motion artifacts are too strong, in which case the motion needs to be estimated from the other phases. The cause of this problem is the width of the phase $T_{w}$, which can be reduced by using smaller rotation times or using dual source CT. However, as we have discussed in Sec. I D, this requires an increase in exposure in order for the minimal required heart rate to remain equal.

\section{IV.D. Minimum required heart rate}

The results in Fig. 11 show that the minimum required heart rate is close to the theoretical limit of $55.1 \mathrm{bpm}$ calculated in Sec. I D. In a volume gap, the voxel data are undefined. Nevertheless, the scanner attempts to fill in the gap; it can be seen that the black vertical lines (the air between the phantom and its guide) are continued in the bands, which suggests interpolation. The fact that the bar in Fig. 11(a) was completely hidden implies that the scanner fills in the bands to some extent, but the data are very noisy and not reliable.

\section{CONCLUSIONS}

We performed experiments to investigate the effect of amplitude and frequency on the detectability of small motions in ECG-gated CT. Also investigated were temporal resolution and minimum required heart rate. The experimental methods can be applied to CT scanners of other manufacturers.
The experiment designed to measure the temporal resolution empirically clearly showed that the duration of each ECG-gated phase is $185 \mathrm{~ms}$ for our scanner and settings, which corresponds to half of the rotation time. The other experiments showed that motions become detectable from amplitudes as small as $0.4 \mathrm{~mm}$ in the $x$ direction and $0.7 \mathrm{~mm}$ in the $z$ direction. Motions up to $2.7 \mathrm{~Hz}$ can be accurately detected. Volume gaps caused by a too low heart rate are expressed in noisy bands in the data that propagate in the $z$ direction. The reconstruction algorithm uses some form of interpolation, but cannot prevent objects in volume gaps becoming hidden.

This study gives insight into the possibilities and limitations for measuring small motions using ECG-gated CT. From the results we conclude that CTA is a suitable technique for studying the expected motions in AAA.

\section{ACKNOWLEDGMENT}

The authors would like to thank Daan van der Vliet for the fruitful discussions.

${ }^{a)}$ Electronic mail: a.klein@ewi.utwente.nl

${ }^{1}$ T. O. Fuchs, M. Kachelriess, and W. A. Kalender, "System performance of multislice spiral computed tomography," IEEE Eng. Med. Biol. Mag. 19, 63-70 (2000).

${ }^{2}$ B. Ohnesorge, T. Flohr, C. Becker, A. F. Kopp, U. J. Schoepf, U. Baum, A. Knez, K. Klingenbeck-Regn, and M. F. Reiser, "Cardiac imaging by means of electrocardiographically gated multisection spiral CT: Initial experience," Radiology 217, 564-571 (2000).

${ }^{3}$ J. Albers, J. M. Boese, C. F. Vahl, and S. Hagl, "In vivo validation of cardiac spiral computed tomography using retrospective gating," Arch. Inst. Pasteur Madagascar 75, 885-889 (2003).

${ }^{4}$ E. E. Williamson, J. Kirsch, P. A. Araoz, W. B. Edmister, D. D. Borgeson, J. F. Glockner, and J. F. Breen, "ECG-gated cardiac CT angiography using 64-MDCT for detection of patent foramen ovale," AJR, Am. J. Roentgenol. 190, 929-933 (2008).

${ }^{5}$ R. Manzke, T. Kohler, T. Nielsen, D. Hawkes, and M. Grass, "Automatic phase determination for retrospectively gated cardiac CT," Med. Phys. 31, 3345-3362 (2004).

${ }^{6}$ M. Dewey, F. Teige, W. Rutsch, T. Schink, and B. Hamm, "CT coronary angiography: Influence of different cardiac reconstruction intervals on image quality and diagnostic accuracy," Eur. J. Radiol. 67, 92-99 (2008). ${ }^{7}$ C. Chartrand-Lefebvre, A. Cadrin-Chenevert, E. Bordeleau, P. Ugolini, R. Ouellet, J. Sablayrolles, and J. Prenovault, "Coronary computed tomography angiography: Overview of technical aspects, current concepts, and perspectives," Can. Assoc. Radiol. J. 58, 92-108 (2007).

${ }^{8}$ M. J. W. Greuter, J. Dorgelo, W. G. J. Tukker, and M. Oudkerk, "Study on motion artifacts in coronary arteries with an anthropomorphic moving heart phantom on an ECG-gated multidetector computed tomography unit," S. Afr. J. Chem. 5, 995-1007 (2005).

${ }^{9}$ B. E. Muhs, K. L. Vincken, J. van Prehn, M. K. Stone, L. W. Bartels, M. Prokop, F. L. Moll, and H. J. Verhagen, "Dynamic Cine-CT angiography for the evaluation of the thoracic aorta. Insight in dynamic changes with implications for thoracic endograft treatment," Parasite 32(5), 532-536 (2006).

${ }^{10}$ A. Teutelink, B. Muhs, K. L. Vincken, L. W. Wartels, S. A. Cornelissen, J. A. van Herwaarden, M. Prokop, F. L. Moll, and H. J. M. Verhagen, "Use of dynamic computed tomography to evaluate pre- and postoperative aortic changes in AAA patients undergoing eneurysm repair," J. Endovasc. Ther. 14(1), 44-49 (2007).

${ }^{11}$ R. Wentz, A. Manduca, J. G. Fletcher, H. Siddiki, R. C. Shields, T. Vrtiska, G. Spencer, A. N. Primak, J. Zhang, T. Nielson, C. McCollough, and L. Yu, "Automatic segmentation and co-registration of gated CT angiography datasets: Measuring abdominal aortic pulsatility," Proc. SPIE 6511, 65111I-8 (2007).

${ }^{12}$ B. E. Muhs, A. Teutelink, M. Prokop, K. L. Vincken, F. L. Moll, and H. J. M. Verhagen, "Endovascular aneurysm repair alters renal artery move- 
ment: A preliminary evaluation using dynamic CTA," J. Endovasc. Ther. 13(4), 476-480 (2006).

${ }^{13}$ A. Klein, W. K. Renema, L. J. Oostveen, L. J. S. Kool, and C. H. Slump, "A segmentation method for stentgrafts in the abdominal aorta from ECG-gated CTA data," Proceedings of SPIE Medical Imaging (SPIE, Bellingham, WA, 2008), Vol. 6916.

${ }^{14}$ T. S. Jacobs, J. Won, E. C. Gravereaux, P. L. Faries, N. Morrissey, V. J. Teodorescu, L. H. Hollier, and M. L. Marin, "Mechanical failure of prosthetic human implants: A 10-year experience with aortic stent graft devices," J. Vasc. Surg. 37(1), 16-26 (2003).

${ }^{15}$ J. E. Roos, J. C. Hellinger, R. Hallet, D. Fleischmann, C. K. Zarins, and G. D. Rubin, "Detection of endograft fractures with multidetector row computed tomography," J. Vasc. Surg. 42, 1002-1006 (2005).

${ }^{16}$ P. Cao, P. D. Rango, G. Parlani, and F. Verzini, "Durability of abdominal aortic endograft with the talent unidoc stent graft in common practice: Core lab reanalysis from the TAURIS multicenter study," J. Vasc. Surg. 49, 859-865 (2009).

${ }^{17}$ S. Demirci, F. Manstad-Hulaas, N. Navab, M. I. Miga, and K. H. Wong, "Quantification of abdominal aortic deformation after EVAR," Medical Imaging 2009: Visualization, Image-Guided Procedures, and Modeling, Lake Buena Vista, FL, February 2009 (SPIE, Bellingham, WA, 2009), Vol. 7261, p. 72611U-9.

${ }^{18}$ K. R. Eide, A. Odegard, H. O. Myhre, and O. Haraldseth, "Initial observations of endovasculair aneurysm repair using Dyna-CT," J. Endovasc. Ther. 14(1), 50-53 (2007).

${ }^{19}$ T. Flohr, B. Ohnesorge, H. Bruder, K. Stierstorfer, J. Simon, C. Suess, and S. Schaller, "Image reconstruction and performance evaluation for ECG-gated spiral scanning with a 16-slice CT system.," Med. Phys. 30, 2650-2662 (2003).
${ }^{20}$ R. Manzke, P. Koken, D. Hawkes, and M. Grass, "Helical cardiac cone beam CT reconstruction with large area detectors: A simulation study," Phys. Med. Biol. 50, 1547-1568 (2005).

${ }^{21}$ A. N. Primak, C. H. McCollough, M. R. Bruesewitz, F. Zhang, and J. G. Fletcher, "Relationship between noise, dose and pitch in cardiac multidetector row CT," Radiographics 26(6), 1785-1794 (2006).

${ }^{22} \mathrm{~S}$. Achenbach, "Half-scan vs. multi-segment reconstruction for computed tomography coronary angiography," Technical Report, Siemens Medical (2006).

${ }^{23}$ D. Ertel, E. Krober, Y. Kyriakou, O. Langner, and W. A. Kalender, "Modulation transfer function-based assessment of temporal resolution: Validation for single- and dual-source CT," Radiology 248, 1013-1017 (2008).

${ }^{24}$ A. Swillens, L. Lanoye, J. D. Backer, N. Stergiopulos, P. R. Verdonck, F. Vermassen, and P. Segers, "Effect of an abdominal aortic aneurysm on wave reflection in the aorta," IEEE Trans. Biomed. Eng. 55, 1602-1612 (2008).

${ }^{25} \mathrm{Z}$. Li and C. Kleinstreuer, "Analysis of biomechanical factors affecting stent-graft migration in an abdominal aortic aneurysm model," J. Biomech. 39, 2264-2273 (2006).

${ }^{26}$ D. Hazer, E. A. Finol, M. Kostrzewa, M. Kopaigorenko, G. Richter, R. Dillmann, X. P. Hu, and A. V. Clough, "Computational biomechanics and experimental validation of vessel deformation based on 4D-CT imaging of the porcine aorta," in Medical Imaging 2009: Biomedical Applications in Molecular, Structural, and Functional Imaging, Lake Buena Vista, FL, February 2009 (SPIE, Bellingham, WA, 2009), Vol. 7262, p. 72621F-9.

${ }^{27}$ W. A. Kalender, Computed Tomography (Publicis Corporate, Erlangen, 2005). 